\title{
Benefits of Using Non-consolidated Domain Influence in Meshless Local Petrov-galerkin (Mlpg) Method for Solving Lefm Problems
}

\author{
Hashim N. Al-Mahmud, Haider K. Mehbes, Ameen A. Nassar* \\ Mechanical Engineering Department, College of Engineering, University of Basrah \\ *Corresponding Author: ameenaledani@yahoo.com
}

Copyright (C) 2014 Horizon Research Publishing All rights reserved.

\begin{abstract}
This paper presents an efficient meshless method in the formulation of the weak form of local Petrov-Galerkin method MLPG. The formulation is carried out by using an elliptic domain rather than conventional isotropic domain of influence. Therefore, the method involves an MLPG formulation in conjunction with an anisotropic weight function. In the elliptic weight function, each node has three characteristic indicated that were major radius, inner radius, and the direction of the local domain. Furthermore, the space that will be covered by the elliptical domain will be less than the area of the circle (isotropic) at the same main diameter. This means leaving many points of integration are not necessary. Therefore, the computational cost will be decreased. MLPG method with the elliptical domain is used in solving problems of linear elastic fracture mechanism LEFM. MATLAB and Fortran codes are used for obtaining the results of this research.The results were compared with those presented in the literature which shows a reduction in the computational cost up to $15 \%$, and an error criteria enhancement up to $25 \%$.
\end{abstract}

Keywords Meshless Methods, Local Petrov-Galerkin Method MLPG, Elliptic Domain

\section{Introduction}

Meshless (MFree) methods, as alternative numerical approaches to eliminate the well-known drawbacks in the finite element and boundary element methods have attracted much attention in the past decade, due to their flexibility, and due to their potential in neglecting the need for the human-labor intensive process of constructing geometric meshes in a domain. There are a number of MFree methods has been developed named according to the technique used in the formulation of the method the major differences in these meshless methods come from the interpolation techniques used [1-4].
In recent decades Mfree methods in computational mechanics have a great attention in solving practical engineering problems in heat transfer, fluid mechanics, and applied mechanics [5-7], especially those problems with discontinuities or moving boundaries. The numerical solution by the traditional finite element method (FEM) of fracture mechanics problems with arbitrary dynamic cracks is limited to simple cases. This is because solution of growing discontinuities requires time consuming remeshing at every time step. For this reason adaptive FEM has become essential. However adaptive remeshing and mapping of variables is a difficult, computationally expensive task and is a source of cumulative numerical errors. The development of meshless methods has enabled the solution of growing cracks without remeshing. Nevertheless, these methods continue to be computationally expensive because of the large nodal densities in meshless methods for an accurate solution. Therefore there is a constant effort to improve the accuracy without increasing the degrees of freedom The main objective of MFree methods is to get rid of or at least alleviate the difficulty of meshing the entire structure ,by only adding or deleting nodes in the entire structure.

A truly meshless method shadow elements are inevitable as in Element-Free Galerkin Methods [8-9]called Meshless Local Petrov-Galerkin Method (MLPG) have been successfully developed in[10-16]for solving linear and non-linear boundary problems[17]. The MLPG method uses local weak forms over a local sub-domain and shape functions from the moving least-squares (MLS) approximation[18]. In the MLS approximation, each node in the global domain $\Omega$ has two sub-domains the $1^{\text {st }}$ is the domain of influence $\Omega_{\mathrm{x}}$, in which a trail function of compact support is used as a weight function. The weight function determines the intensity of the effect of a node at various points in its domain of influence, the $2^{\text {nd }}$ is a sub-domain for the test function $\Omega_{\mathrm{s}}$ (Integral Domain) which often similar in shape but smaller than the trial function. These nodal trial and test functions are centered with maximum value at the nodes (which are the centers of the domains $\Omega_{\mathrm{x}}$ and $\Omega_{\mathrm{s}}$ ), 
respectively, and, in general, vanish at the boundaries $\partial \Omega_{\mathrm{x}}$ and $\partial \Omega_{\mathrm{s}}$ of $\Omega_{\mathrm{x}}$ and $\Omega_{\mathrm{s}}$, respectively having a bell/tent shape. The local domains $\Omega_{\mathrm{x}}$ and $\Omega_{\mathrm{s}}$ can be of arbitrary shapes, such as circle, and square rectangle (conventional domains) in $2 \mathrm{D}$ geometries as shown in Figures (1-a,b and c), and spheres, square or rectangular parallelepipeds in $3 \mathrm{D}$ geometries [19-20]. The sizes of $\Omega_{\mathrm{x}}$ and $\Omega_{\mathrm{s}}$ can be arbitrary, different from each other, and different for each node, in general. Essential boundary conditions are enforced while using the meshless approximations, approximately by using a penalty formulation and Lagrange multipliers [1].

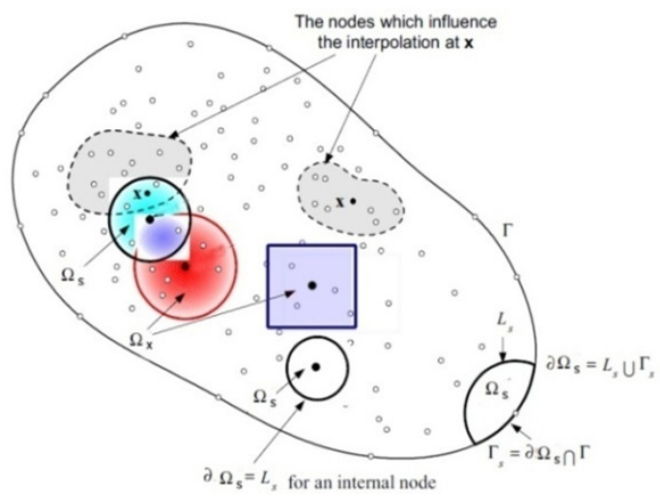

Figure (1-a). Schematics of the MLPG method [1]

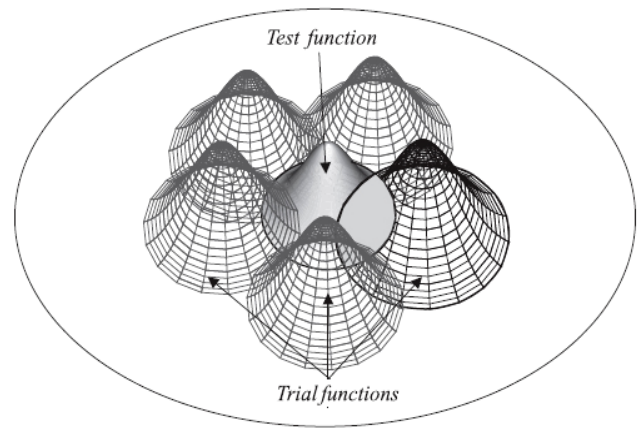

Figure (1-b). Schematics of the trial and test functions in $\Omega$ [5].

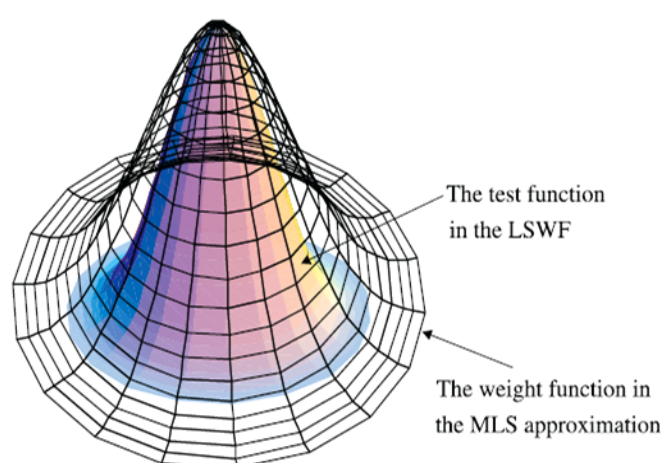

Figure (1-c). The test function in the Local Symmetric Weak Form (LSWF) and the weight function in the MLS approximation[11]
MLPG, accuracy and effectiveness are dependent on the nodal domain of influence and type of the weight function. In this work, non-consolidated (anisotropic) weight function in the elliptic form is introduced to improve the efficiency of the MLPG with an anisotropic support in some problems. In the using non-consolidated weight functions, the influence domain of each node may vary with direction. As a consequence, the definition of the influence domain based on non-consolidated weight function, improves the numerical efficiency of MLPG. In such case, the influence domain of each node can be determined so that the nodal overlapping decreases. Thus, good results can be achieved with less computational efforts.

\section{MLS Approximation}

It has been shown that moving least-square methods and weight functions share many features for the constructing the approximation of the solution. In the MLS technique that presented by [18], the approximation $u^{h}(X)$ is expressed as the inner product of a vector of the polynomial basis $P(X)$ and a vector of the coefficient $a(x)$.

$$
u^{h}(X)=P^{T}(X) a(X)=\sum_{i=1}^{m} P_{i}(X) a_{i}(X)
$$

where $m$ denotes the number of terms in the basis. In two dimensions a complete polynomial basis of order $\mathrm{m}$ is given by

$$
P^{T}(x, y)=\left(1, x, y, x^{2}, x y, y^{2}, \ldots, x^{m}, \ldots, x^{m} y^{m-k}, \ldots, y^{m}\right)
$$

For bi-linear, the basis function is considered as

$$
P^{T}(x, y)=(1, x, y, x y), m=4
$$

Also, the linear basis is provided by

$$
P^{T}(x, y)=(1, x, y), m=3
$$

Vector of unknown parameters that depends on $x$ is given as

$$
a(X)=\left(a_{1}(x), a_{2}(x), \ldots, a_{m}(x)\right)
$$

These basis functions are not required to be polynomials as shown in equations (3) and (4). For problems involving cracks, a convenient way of capturing $1 / \sqrt{r}$ stress-singularity in linear-elastic fracture mechanics is calculated by using [2]

$$
P^{T}(x, y)=(1, x, y, \sqrt{r}), m=4
$$

or

The nodal influence domain is usually considered having a consolidated form in the shape of a circle or sphere. In the

$$
P^{T}(x, y)=(1, x, y, \sqrt{r} \cos (\theta / 2), \sqrt{r} \sin (\theta / 2), \sqrt{r} \sin (\theta / 2) \sin \theta, \sqrt{r} \cos (\theta / 2) \sin \theta), m=7
$$


Where $r$ and $\theta$ are polar coordinates with the crack tip as the origin. Equations (6) and (7) represent fully enriched and partially enriched basis functions, respectively.

In equation (1), the coefficient vector, $a(x)$ is determined by minimizing a weighted discrete $\mathrm{L}_{2}$ norm, defined as

$$
J(X)=\sum_{I=1}^{n} w\left(X-X_{I}\right)\left[P^{T}\left(X_{I}\right) a(X)-u_{I}\right]^{2}
$$

Where $\mathrm{n}$ is the number of nodes $I$ such that $w\left(x-x_{I}\right) \succ 0$; $w_{I}(x)=w\left(x-x_{I}\right)$ is the weight function associated with node $I$ which is non-zero over a limited support called the influence domain of node $I$.

Equation ( 8) can also be written as [1]

$$
J(X)=[P . a(X)-u]^{T} W[P . a(X)-u]
$$

Where

$$
\begin{gathered}
u^{T}=\left\{u_{1}, u_{2}, \ldots, u_{n}\right\} \\
P=\left[\mathrm{P}_{\mathrm{ij}}\right]_{n \times m}=\left[\begin{array}{cccc}
1 & x_{1} & y_{1} & x_{1} y_{1} \\
1 & x_{2} & y_{2} & x_{2} y_{2} \\
\vdots & \vdots & \vdots & \vdots \\
1 & x_{n} & y_{n} & x_{n} y_{n}
\end{array}\right] \\
W(X)=\left(w_{i j}\right)_{n \times n}=\left[\begin{array}{ccccc}
w\left(X-X_{1}\right) & 0 & \cdots & 0 \\
0 & w\left(X-X_{2}\right) & \cdots & 0 \\
\vdots & & \ddots & \\
0 & 0 & \cdots & w\left(X-X_{n}\right)
\end{array}\right]
\end{gathered}
$$

The stationarity of $J(X)$ with respect to $a(X)$ yields

$$
\frac{\partial J}{\partial a}=A(X) a(X)-B(X) u=0
$$

Hence

$$
\begin{gathered}
a(X)=A^{-1}(X) B(X) u \\
A(X)=P^{T} W(X) P \\
=w\left(X-X_{I}\right)\left[\begin{array}{cccc}
1 & x_{1} & y_{1} & x_{1} y_{1} \\
x_{1} & x_{1}^{2} & x_{1} y_{1} & x_{1}^{2} y_{1} \\
y_{1} & y_{1} x_{1} & y_{1}^{2} & x_{1} y_{1}^{2} \\
x_{1} y_{1} & x_{1}^{2} y_{1} & x_{1} y_{1}^{2} & x_{1}^{2} y_{1}^{2}
\end{array}\right]+\cdots+ \\
w\left(X-X_{n}\right)\left[\begin{array}{cccc}
1 & x_{n} & y_{n} & x_{n} y_{n} \\
x_{n} & x_{n}^{2} & x_{n} y_{n} & x_{n}^{2} y_{n} \\
y_{n} & y_{n} x_{n} & y_{n}^{2} & x_{n} y_{n}^{2} \\
x_{n} y_{n} & x_{n}^{2} y_{n} & x_{n} y_{n}^{2} & x_{n}^{2} y_{n}^{2}
\end{array}\right] \\
B(X)=P^{T} W(X)
\end{gathered}
$$

$$
=\left[\begin{array}{llll}
w\left(X-X_{I}\right) P\left(X_{1}\right) & w\left(X-X_{2}\right) P\left(X_{2}\right) & \cdots & w\left(X-X_{n}\right) P\left(X_{n}\right)
\end{array}\right]
$$

The MLS approximants can be defined as

$$
u^{h}(X)=\sum_{I=1}^{n} \varphi_{I}(X) u_{I}=\Phi(X) u
$$

Where the shape function $\Phi_{I}(X)$ is

$$
\Phi_{I}(X)=P^{T}(X) A^{-1}(X) B_{I}(X)
$$

The partial derivatives of $\Phi_{I}(X)$ can be obtained as follows

$$
\Phi_{I, i}(X)=\sum_{j=1}^{m}\left\{p_{j, i}\left(A^{-1} B\right)_{j I}+p_{j}\left(A_{, i}^{-1} B+A^{-1} B_{, i}\right)_{j I}\right\}
$$

Where

$$
A_{, i}^{-1}=-A^{-1} A_{, i} A^{-1}
$$

$\operatorname{In}$ which ()$_{, i}=\frac{\partial()}{\partial x_{i}}$.

\section{The MLPG Weak Formulation}

Consider the following two-dimensional elasto-statics problem on the domain $\Omega$ bounded by the boundary $\Gamma[1,10]$ :

$$
\sigma_{i j}, j+b_{i}=0 \text {, in } \Omega \text {, }
$$

where $\sigma_{i j}$ is the stress tensor, $b_{i}$ is the body force, a repeated index implies summation over the range of the index, the rectangular Cartesian coordinates and employing the boundary conditions gives;

$$
\begin{gathered}
u_{i}=\bar{u}_{i} \text { on } \Gamma_{\mathrm{u}} \\
\mathrm{t}_{\mathrm{i}} \equiv \sigma_{\mathrm{ij}} \mathrm{n}_{\mathrm{j}}=\overline{\mathrm{t}}_{\mathrm{i}} \text { on } \Gamma_{\mathrm{t}}
\end{gathered}
$$

where $\bar{u}_{i}$ and $\bar{t}_{\mathrm{i}}$ are the prescribed displacements and tractions, respectively, on the boundary $\Gamma_{\mathrm{u}}$ and the boundary $\Gamma_{t}$, and $n_{j}$ is the unit outward normal to the boundary $\Gamma . \Gamma_{u}$ and $\Gamma_{\mathrm{t}}$ are complementary subsets of $\Gamma$.

A generalized local weak form of eq. (21) and Eqs. (22),(23) over a local sub-domain $\Omega s$ can be written as follows [1]:

$$
\int_{\Omega_{S}}\left(\sigma_{i j, j}+b_{i}\right) v_{\mathrm{i}} d \Omega-\int_{\Gamma_{s u}} \alpha\left(u_{i}-\bar{u}_{i}\right) v_{\mathrm{i}} d \Gamma=0
$$

where $u_{i}$ and $v i$ are the trial and the test functions, respectively, and $\Gamma s u$ is the part of theboundary $\partial \Omega s$ over which essential boundary conditions are specified. In general, $\partial \Omega s=\Gamma s \cup L s$ with $\Gamma s$ being the part of the local boundary located on the global boundary and $L s$ being the other part of local boundary over which no boundary condition is specified, i.e., $\Gamma s=\partial \Omega s \cap \Gamma$ and $\Gamma s=\partial \Omega s L s$ see Figure (1-a) . In eq. (24) $\boldsymbol{\alpha}$ is a penalty parameter ( $\boldsymbol{\alpha}>>$ Young's 
modulus/Length ) which is used to impose the essential boundary conditions. Also, the dimensions of $\boldsymbol{\alpha}$ are such that the two terms in eq. (24) have the same units. $\boldsymbol{\alpha}$ could be a function of $\mathbf{x}$ but is usually taken to be a constant.

Using $\sigma_{i j, j} v_{i}=\left(\sigma_{i j} v_{i}\right)_{, j}-\sigma_{i j} v_{i, j}$ and the divergence theorem in eq. (24) leads to

$$
\begin{gathered}
\int_{\partial \Omega_{S}} \sigma_{i j} \mathrm{n}_{\mathrm{j}} v_{\mathrm{i}} d \Gamma-\int_{\Omega_{S}}\left(\sigma_{i j} v_{i, j}-b_{i} v_{i}\right) d \Omega-\alpha \int_{\Gamma_{s u}}\left(u_{i}-\right. \\
\text { uivi } d \Gamma=0
\end{gathered}
$$

Applying the natural boundary condition gives;

$$
\begin{array}{r}
\int_{L_{s}} t_{i} v_{i} d \Gamma+\int_{\Gamma_{s u}} t_{i} v_{i} d \Gamma+\int_{\Gamma_{s t}} \bar{t}_{i} v_{i} d \Gamma-\int_{\Omega_{S}}\left(\sigma_{i j} v_{i, j}-\right. \\
\text { bi vi } d \Omega-\alpha \Gamma \text { suui-uivi } d \Gamma=0
\end{array}
$$

In order to simplify equation (26), the test functions $v i$ are chosen such that they vanish on $L s$. This can be accomplished by using the weight function wi in the MLS approximation as also the test function $v i$, but the radius $r i$ of the support of the weight function is replaced by the radius $r o$ of the local domain $\Omega s$. Using these test functions and rearranging eq. (26) gives the Local Symmetric weak form (LSWF);

$$
\begin{gathered}
\int_{\Omega_{S}} \sigma_{i j} v_{i, j} d \Omega+\alpha \int_{\Gamma_{s u}} u_{i} v_{\mathrm{i}} d \Gamma-\int_{\Gamma_{s u}} t_{i} v_{i} d \Gamma \\
=\int_{\Gamma_{s t}} \bar{t}_{i} v_{i} d \Gamma \\
+\alpha \int_{\Gamma_{s u}} \bar{u}_{i} v_{\mathrm{i}} d \Gamma+\int_{\Omega_{S}} b_{i} v_{i} d \Omega
\end{gathered}
$$

For 2-D problems, two independent sets of test functions should be applied in eq. (27) which gives

$$
\begin{gathered}
\int_{\Omega_{S}} \sigma_{i j} v_{k i, j} d \Omega+\alpha \int_{\Gamma_{s u}} u_{i} v_{\mathrm{ki}} d \Gamma-\int_{\Gamma_{s u}} t_{i} v_{k i} d \Gamma \\
=\int_{\Gamma_{s t}} \bar{t}_{i} v_{k i} d \Gamma \\
+\alpha \int_{\Gamma_{s u}} \bar{u}_{i} v_{\mathrm{ki}} d \Gamma+\int_{\Omega_{S}} b_{i} v_{k i} d \Omega
\end{gathered}
$$

where $v k i$ is the $i$ th component of the $k$ th test function. For simplicity, eq. (28) can be written in matrix form as:

$$
\begin{array}{r}
\int_{\Omega_{\mathrm{S}}} \varepsilon_{\mathrm{v}} \sigma \mathrm{d} \Omega+\alpha \int_{\Gamma_{\mathrm{su}}} \mathrm{vud} \Gamma-\int_{\Gamma_{\text {su }}} \mathrm{v} \mathrm{t} \mathrm{d} \Gamma=\int_{\Gamma_{\mathrm{st}}} \mathrm{v} \overline{\mathrm{t}} \mathrm{d} \Gamma+ \\
\alpha \int_{\Gamma_{\mathrm{su}}} \mathrm{v} \overline{\mathrm{u}} \mathrm{d} \Gamma+\int_{\Omega_{\mathrm{S}}} \mathrm{v} \mathrm{b} \mathrm{d} \Omega
\end{array}
$$

where $\varepsilon v$ denotes the strain matrix derived from the test functions, and $\sigma$ is the stress vector derived from the trial functions. That is,

$$
\begin{gathered}
\sigma=\left\{\begin{array}{l}
\sigma_{11} \\
\sigma_{22} \\
\sigma_{12}
\end{array}\right\} \equiv \\
{\left[\begin{array}{l}
\sigma_{x x} \\
\sigma_{y y} \\
\sigma_{x y}
\end{array}\right\}, \boldsymbol{\varepsilon}_{v}=\left[\begin{array}{lll}
\varepsilon_{11}^{(1)} & \varepsilon_{22}^{(1)} & \gamma_{12}^{(1)} \\
\varepsilon_{11}^{(2)} & \varepsilon_{22}^{(2)} & \gamma_{12}^{(2)}
\end{array}\right] \equiv} \\
{\left[\begin{array}{lll}
\varepsilon_{x x}^{(1)} & \varepsilon_{y y}^{(1)} & \gamma_{x y}^{(1)} \\
\varepsilon_{x x}^{(2)} & \varepsilon_{y y}^{(2)} & \gamma_{x y}^{(2)}
\end{array}\right]}
\end{gathered}
$$

where the superscript $i$ denotes the $i$ th test function. Functions $\mathbf{v}, \mathbf{u}, \mathbf{t}$, and $\mathbf{b}$ are defined as follows:

$$
\mathrm{v}=\left[\begin{array}{ll}
v_{11} & v_{12} \\
v_{21} & v_{22}
\end{array}\right], \mathrm{u}=\left\{\begin{array}{l}
u_{1} \\
u_{2}
\end{array}\right\}, \mathrm{t}=\left\{\begin{array}{l}
t_{1} \\
t_{2}
\end{array}\right\}, \mathrm{b}=\left\{\begin{array}{l}
b_{1} \\
b_{2}
\end{array}\right\}
$$

The two sets of test functions $\mathbf{v}$ in eq. (31) should be linearly independent. The simplest choice for $\mathrm{v}$ is

$$
v_{\mathrm{ij}}=v \delta_{\mathrm{ij}} \text { or } \mathrm{v}=v \mathrm{I},
$$

where $\delta_{i j}$ is the Kronecker delta and I is the identity matrix . As long as the union of all local sub-domains covers the global domain, the equilibrium equations (21) and the boundary conditions (22),(23) will be satisfied in the global domain $\Omega$ and on its boundary $\Gamma$ respectively.

\section{Discretization of the Weak Form}

Substituting the MLS approximation eq. (17) into eq. (29) and summing over all nodes leads to the following discredited system of linear equations:

$$
\begin{aligned}
& \sum_{\mathrm{j}=1}^{\mathrm{n}} \int_{\Omega_{\mathrm{S}}} \varepsilon_{\mathrm{v}}\left(\mathrm{x}, \mathrm{x}_{\mathrm{i}}\right) \mathrm{DB}_{\mathrm{j}} \mathrm{u}_{\mathrm{j}} \mathrm{d} \Omega+ \\
& \alpha \sum_{\mathrm{j}=1}^{\mathrm{n}} \int_{\Gamma_{\mathrm{su}}} \mathrm{v}\left(\mathrm{x}, \mathrm{x}_{\mathrm{i}}\right) \mathrm{S} \phi_{\mathrm{j}} \mathrm{u}_{\mathrm{j}} \mathrm{d} \Gamma- \\
& \sum_{\mathrm{j}=1}^{\mathrm{n}} \int_{\Gamma_{\mathrm{su}}} \mathrm{v}\left(\mathrm{x}, \mathrm{x}_{\mathrm{i}}\right) \mathrm{NDSB}_{\mathrm{j}} \mathrm{u}_{\mathrm{j}} \mathrm{d} \Gamma=\int_{\Gamma_{\mathrm{st}}} \mathrm{v}\left(\mathrm{x}, \mathrm{x}_{\mathrm{i}}\right) \overline{\mathrm{t}} \mathrm{d} \Gamma+ \\
& \alpha \int_{\Gamma_{\text {su }}} \mathrm{v}\left(\mathrm{x}, \mathrm{x}_{\mathrm{i}}\right) \mathrm{Su} \mathrm{d} \Gamma+\int_{\Omega_{\mathrm{S}}} \mathrm{v}\left(\mathrm{x}, \mathrm{x}_{\mathrm{i}}\right) \mathrm{b} \mathrm{d} \Omega \text { (32) }
\end{aligned}
$$

where $\mathrm{v}(\mathrm{x}, \mathrm{x} i)$ is the value at $\mathbf{x}$ of the test function, corresponding to node $i$, and

$$
\begin{gathered}
\mathrm{N}=\left[\begin{array}{ccc}
n_{1} & 0 & n_{2} \\
0 & n_{2} & n_{1}
\end{array}\right] \\
\mathrm{B}_{j}=\left[\begin{array}{cc}
\phi_{j, 1} & 0 \\
0 & \phi_{j, 2} \\
\phi_{j, 2} & \phi_{j, 1}
\end{array}\right] \\
\mathrm{D}=\bar{E} /\left(1-\bar{v}^{2}\right)\left[\begin{array}{ccc}
1 & \bar{v} & 0 \\
\bar{v} & 1 & 0 \\
0 & 0 & (1-\bar{v}) / 2
\end{array}\right]
\end{gathered}
$$

We note that

$$
\begin{gathered}
\overline{\mathrm{E}}=\left\{\begin{array}{c}
\mathrm{E} \\
\mathrm{E} /\left(1-v^{2}\right)
\end{array}, \quad \bar{v}=\left\{\begin{array}{c}
v \\
v /(1-v),
\end{array},\right. \text { for plane stress }\right. \\
\mathbf{S}=\left[\begin{array}{cc}
\mathrm{S}_{1} & 0 \\
0 & \mathrm{~S}_{2}
\end{array}\right], S_{i}=\left\{\begin{array}{c}
1 \quad \text { if } u_{i} \text { is prescribed on } \Gamma_{u}, \\
0, \text { if } u_{i} \text { is not prescribed on } \Gamma_{u} .
\end{array}\right.
\end{gathered}
$$

Eq. (32) can be simplified into the following system of linear algebraic equations in $\mathbf{u}_{\mathrm{j}}$

$$
\sum_{j=1}^{N} K_{i j} U_{j}=f_{i} \quad i=1,2, \ldots . N
$$

where $N$ is the total number of nodes. The so-called "stiffness" matrix $\mathrm{K}$ and the "load" vector $\mathbf{f}$ are defined by

$$
\begin{aligned}
& \mathrm{K}_{\mathrm{ij}}=\int_{\Omega_{\mathrm{s}}} \varepsilon_{\mathrm{v}}\left(\mathrm{x}, \mathrm{x}_{\mathrm{i}}\right) D B_{\mathrm{j}} \mathrm{d} \Omega+\alpha \int_{\Gamma_{\mathrm{su}}} \mathrm{v}\left(\mathrm{x}, \mathrm{x}_{\mathrm{i}}\right) \mathrm{S} \phi_{\mathrm{j}} \mathrm{d} \Gamma- \\
& \int_{\Gamma_{\mathrm{su}}} \mathrm{v}\left(\mathrm{x}, \mathrm{x}_{\mathrm{i}}\right) \mathrm{NDB}_{\mathrm{j}} \mathrm{S} \mathrm{d} \Gamma \\
& \int_{\Gamma_{s t}} \mathrm{v}\left(\mathrm{x}, \mathrm{x}_{\mathrm{i}}\right) \overline{\mathrm{t}} \mathrm{d} \Gamma+\alpha \int_{\Gamma_{\mathrm{su}}} \mathrm{v}\left(\mathrm{x}, \mathrm{x}_{\mathrm{i}}\right) S \overline{\mathrm{u}} \mathrm{d} \Gamma+\int_{\Omega_{\mathrm{S}}} \mathrm{v}\left(\mathrm{x}, \mathrm{x}_{\mathrm{i}}\right) \mathrm{b} \mathrm{d} \Omega
\end{aligned}
$$

\section{The Influence of Domain Form}

The shape functions $\theta_{\mathrm{i}}$ are obtained from the window 
functions, these functions have influence domain of the node. The domain size is defined by the so called dilatation parameter or smoothing length. It is critical to solution accuracy, stability and plays the role of the element size in the finite element method. The final characteristics of weight functions are its functional forms. The weight function should be continuous and positive in its support. Some commonly used weight functions are the Gaussian weight function and the quartic spline weight function[10]. They are given, respectively in equation (41) and (42).

$$
\begin{gathered}
\left.w_{i}(\mathbf{x})=\left\{\frac{\exp \left[-\left(d_{i} / c_{i}\right)^{2 k}\right]-\exp \left[-\left(r_{i} / c_{i}\right)^{2 k}\right]}{1-\exp \left[-\left(r_{i} / c_{i}\right)^{2 k}\right]}\right\}, \begin{array}{l}
0 \leq d_{i} \leq r_{i} \\
0
\end{array}\right\}, \begin{array}{c}
d_{i} \geq r_{i} \\
w_{i}(\mathbf{x})=\left\{\begin{array}{c}
1-6\left(\frac{d_{i}}{r_{i}}\right)^{2}+8\left(\frac{d_{i}}{r_{i}}\right)^{3}-3\left(\frac{d_{i}}{r_{i}}\right)^{4} \\
0
\end{array}\right\}, \quad \begin{array}{c}
0 \\
0
\end{array} \quad d_{i} \leq r_{i} \geq r_{i}
\end{array}
\end{gathered}
$$

Here $d i=\left|\mathrm{x}-\mathrm{x}_{\mathrm{i}}\right|$ is the distance from the sampling point $\mathbf{x}$ to the node $\mathrm{x} i$, and $r i$ is the radius of the domain of influence for the weight function wi. The parameters $c i$ and $k$ in eq.(41) control the shape of the Gaussian weight function wi. The parameter $k$ can be taken as 1 in eq. (41). So far, there is no theory to determine an optimal value of the parameter $c i$ and is chosen empirically. It is suggested that $c i$ defined as the distance from node $\mathbf{x} i$ to the third nearest neighboring node. The domain of influence $r i$ can be chosen as $\mathrm{r}_{\mathrm{i}} / \mathrm{c}_{\mathrm{i}} \geq 3.5$ so that the weight function wi covers sufficientAlso, it should be small enough to retain the local characteristic of the approximationnumber of nodes to ensure the non-singularity of $\mathrm{A}$ in eq.(18) see Figure (2-a).

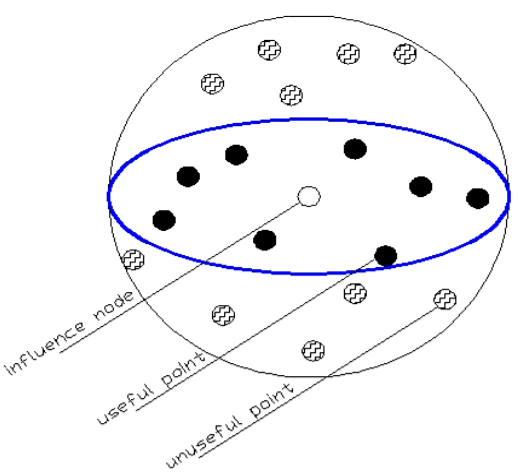

Figure (2-a).

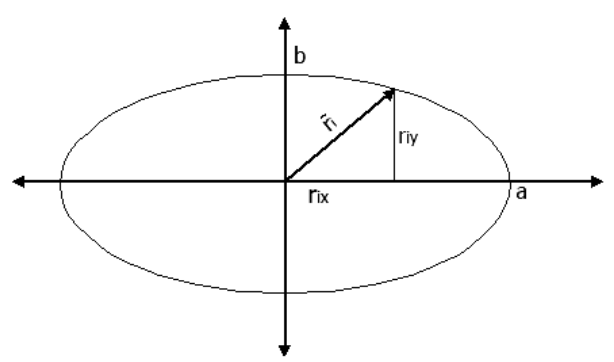

Figure (2-b).

Figure 2. Requirement Representation of Elliptic Domain, and Circular Domain to the Integrations Points
In elliptic weight functions, each node has a major radius of influence $a$, a minor radius of influence $b$ (where $\mathrm{a}=\mathrm{b}$ for circular domain ) and an angle $\theta$ that determines the direction of the maximum radius of the influence ellipse. The influence elliptic domain of a node is determined by three parameters in contrast to one in the circular domain weights. The availability of more controlling parameters for determination of the influence domain of each node helps to increase computational efficiency of MLPG method. The radius of influence of the circular domain $r_{i}$ is modified to the elliptic form $\bar{r}_{\mathrm{i}}$

$$
\left(\frac{r_{i x}}{a}\right)^{2}+\left(\frac{r_{i y}}{b}\right)^{2}=1
$$

Also,, it can be write in general form:

$$
\bar{r}_{i}=\sqrt{r_{i x}{ }^{2}+r_{i y}{ }^{2}}
$$

Where $\mathrm{r}_{\mathrm{ix}}, \mathrm{r}_{\mathrm{iy}}$ are a normalized horizontal and vertical distance from the sampling point $\mathbf{x}$ to the node $\mathbf{x} i$ on $\partial \Omega s$ respectively, see Figure (2-b).

\section{The MLPG Solution Algorithm}

- MODELING: constructing the problem domain (nodal configuration).

- INPUT: provides the data required for running the solution.

- DMAT: creates the $3 \times 3$ constitutive D-matrix.

- GAUS: locates the Gaussian integration points in the natural plane.

- INFLUD: calculates the domain of influence and domain of integration for every node.

- XYQPO: calculates the sub-domain portion of the stiffness $\mathrm{K}$.

- WCOUNT: calculates number of nodes whose weight functions $w\left(\mathbf{x}_{\mathrm{i}}\right)>0$.

- TESTFUN: calculates the value of the test function $\mathrm{v}_{i}$ for the given influence node.

- $\mathrm{ABM}$ : calculates the matrix $\mathrm{A}$, the matrix $\mathrm{B}$ and their derivatives.

- PVE: calculates the basis vectors and their $\mathrm{x}, \mathrm{y}$ derivatives.

- SHAPE: calculates the shape function $\phi_{i}$ and its $\mathrm{x}, \mathrm{y}$ derivatives.

- NSTIFF: performs the numerical integration for the stiffness $\mathrm{K}$ over the nodal domain.

- BCAPLY: performs the numerical integration of the load vector $\mathrm{f}$.

- BKCAL: performs the numerical integration of the boundary portion of the stiffness matrix $\mathrm{K}$.

- OUTPUT: is for the output of displacements and stresses over the whole domain.

\section{Numerical Results}

Example 1. This example involves the Timoshenko beam; 
consider a beam of dimensions $L \times D$, subjected to a parabolic traction at the free end as shown in Figure (3) . The beam is considered to be of unit depth and is in plane stress state. This problem was numerically solved by MLPG with conventional domains previously by $[1,21]$. The parabolic traction is given by

$$
t_{y}(y)=-\frac{P}{2 I}\left(\frac{D^{2}}{4}-y^{2}\right)
$$

Where $I=D^{3} / 12$ is the second moment of area. The exact displacement solution and exact stresses for this problem are

$$
\begin{gathered}
u_{x}(x, y)=-\frac{P y}{6 E I}\left[(6 L-x) x+(2+v)\left(y^{2}-\frac{D^{2}}{4}\right)\right] \\
u_{y}(x, y)=\frac{P y}{6 E I}\left[3 v y^{2}(L-x)+(4+5 v) \frac{D^{2} x}{4}+(3 L-x) x^{2}\right.
\end{gathered}
$$

$$
\begin{gathered}
\sigma_{x}(x, y)=-\frac{P(L-x) y}{I} \\
\sigma_{x y}(x, y)=-\frac{P}{2 I}\left(\frac{D^{2}}{4}-y^{2}\right) \\
\sigma_{y}(x, y)=0
\end{gathered}
$$

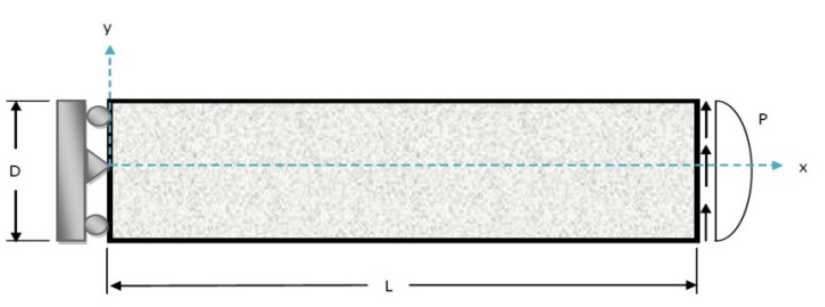

Figure 3. Cantilever beam under an end load.

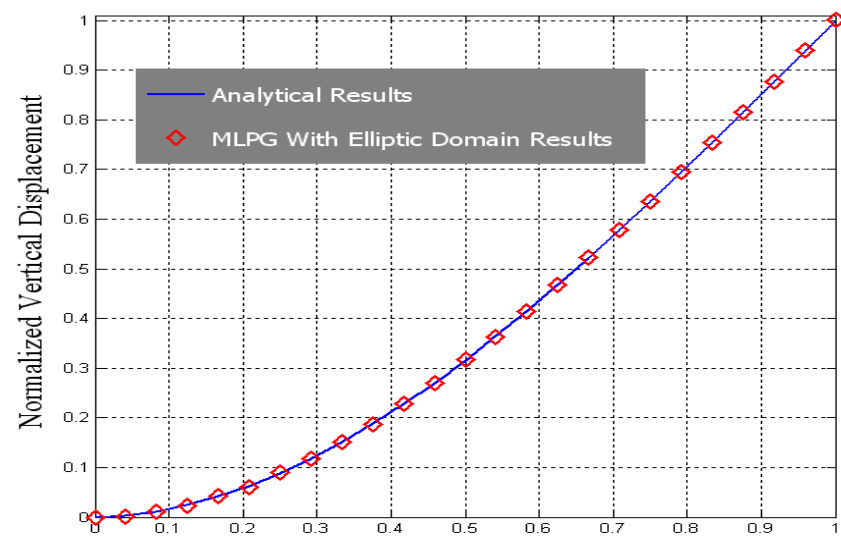

$\mathrm{x} / \mathrm{L}$

Figure 4. Normalized vertical displacement of a cantilever beam under an end loading

In this paper, the parameters for cantilever beam are material properties as $E=1, v=0.25$ and the beam dimensions are $D=4$ and $L=24$. Shear force is $P=1$. Regular uniform nodal configurations with number of nodes are 100,115 , and 125 . At the circular domain, the problem is solved by using the MLS approximation, with a support size of $(2.3 \times$ nodal distance $)$, and a test size of $(0.6 \times$ nodal distance $)$. While in the anisotropic domain, elliptical nodal support of radius 2.3 times from longer nodal spacing and 1.95 from smaller nodal spacing are employed in $\mathrm{x}$ and $\mathrm{y}$ direction respectively.

In Table 1, the vertical displacement in the end of beam that is calculated by MLPG is compared with the exact solution at three nodal configurations. By the error criteria, this table shows excellent agreement between MLPG and the analytical solution, particularly in the results of the elliptic domain.

Table 1. Comparsion of Error at Vertical Displacement End of Beam

\begin{tabular}{|c|c|c|}
\hline \multirow{2}{*}{ Nodes } & \multicolumn{2}{|c|}{ Error \% } \\
\cline { 2 - 3 } & Elliptic Domain & Circular Domain \\
\hline $25 \times 5$ & 0.25 & 0.33 \\
\hline $23 \times 5$ & 2.31 & 2.64 \\
\hline $20 \times 5$ & 2.60 & 2.75 \\
\hline
\end{tabular}

Furthermore, reduction of computational cost is verified. Table 2 clears that the time of computation in the elliptic domain is less than the time of computation in the circle domain. This is due to less value of nodes in Equation (8) when the elliptic domain is used.

Table 2. Comparsion of Time Cost of Elliptic and Circle Domain

\begin{tabular}{|c|c|c|}
\hline \multirow{2}{*}{ Nodes } & \multicolumn{2}{|c|}{ Time of compution (time unit) } \\
\cline { 2 - 3 } & Elliptic Domain & Circular Domain \\
\hline $25 \times 5$ & 3.0292 & 3.5541 \\
\hline $23 \times 5$ & 2.882 & 3.0101 \\
\hline $20 \times 5$ & 2.3788 & 2.4343 \\
\hline
\end{tabular}

Example 2. Figure (5-a) displays a rectangular plate with a central crack loaded by an axial tensile traction. Basic parameters are $\mathrm{L}=52 \mathrm{~mm}, \mathrm{D}=20 \mathrm{~mm}, \bar{t}=0.4 \mathrm{H}(\mathrm{t}) \mathrm{Gpa}, \mathrm{E}=76$ $\mathrm{GPa}$, poisson's ratio $v=0.286, \alpha=10^{7} \mathrm{MPa} / \mathrm{m}$ density $\rho=2450 \mathrm{Kg} / \mathrm{m} 3$ and $\mathrm{a}=12 \mathrm{~mm}$.

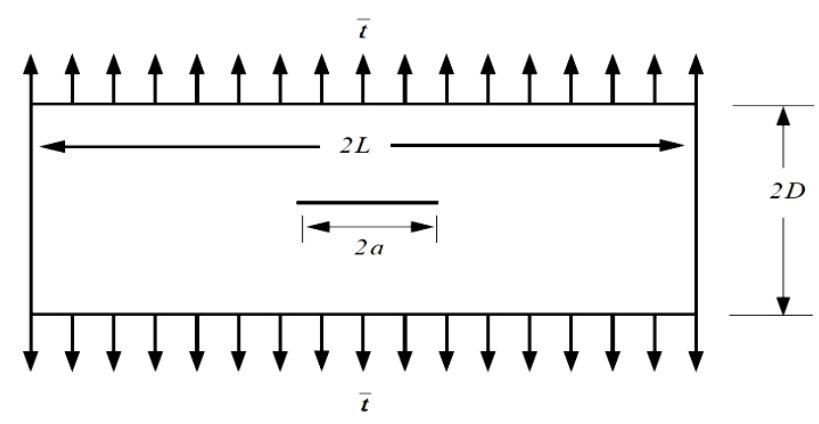

Figure 5-a. A schematic sketch of the centrally cracked plate 
A plane strain state of deformation is supposed in computing. Due to the symmetry of the problem about two centroidal axes, only a quadrant of the plate is considered and discretized using 2534 nodes see Figure (5-b) . 9×9 Gauss points are used in a sub-domain $\Omega_{\mathrm{s}}, 9$ Gauss points are used on each section of $L_{s}$ and $\Gamma_{s}$ for numerical integration. The enrichment of nodes at crack tip can be cleared in Figure (5-b). In the MLS approximation, the linear basis function and the Gaussian weight function for both circular and elliptic sub-domains $\Omega_{\mathrm{s}}$ are used. The present results, using elliptical sub-domains $\Omega_{\mathrm{s}}$ with a major radius of influence $\mathrm{a}=$ $r_{i}$, minor radius of influence $b=0.75 r_{i}$ and an angle $\theta=0$ that determines the direction of the maximum radius of the influence ellipse, are showing in Figures $(6,7$, and 8).

These Figures set the variation of stresses $\boldsymbol{\sigma}_{\mathbf{x}}, \boldsymbol{\sigma}_{\mathbf{y}}$ and $\boldsymbol{\sigma}_{\mathbf{x y}}$ with the distance to the tip is made at a certain constant time. Results of an anisotropic domain (elliptic domain) have a good agreement compared with the results of reference [22] which is obtained using a circular domains $\Omega_{\mathrm{s}}$, where (a) equals (b) for circular domain, in addition the formulation here is more efficient as it demonstrated in example one. Finally, The ratio of the elapsed time for solving the problem with elliptic domain to circular domain is equal to 0.742 .

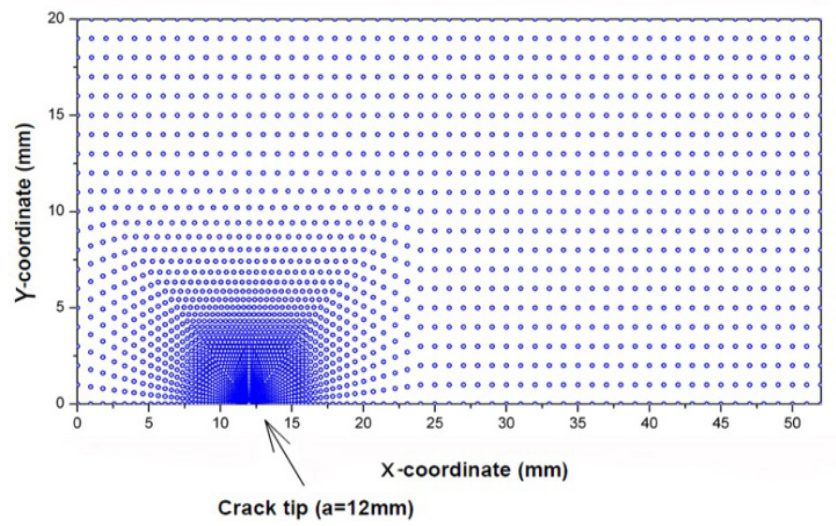

Figure 5-b. The nodal mesh for one quarter of the centrally cracked plate

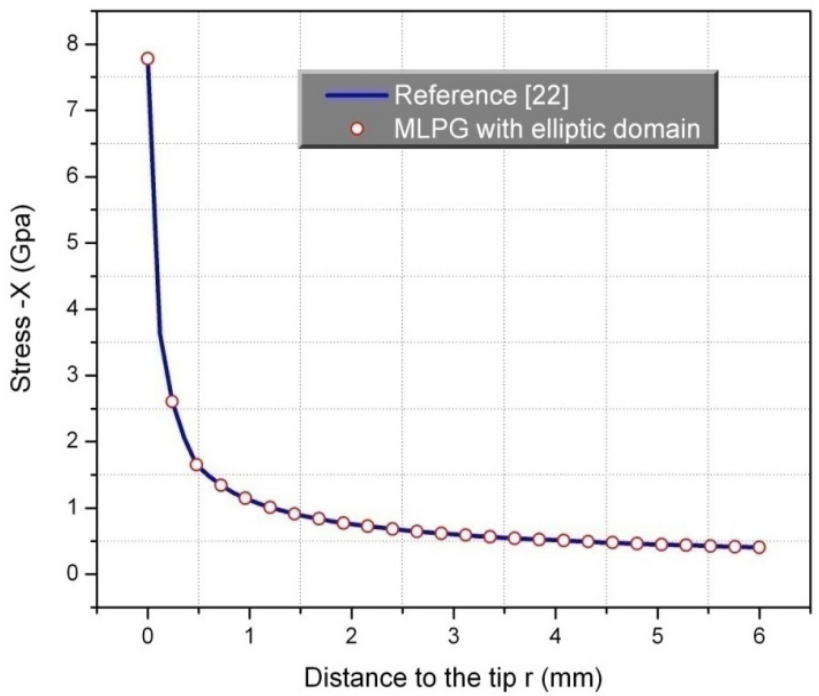

Figure 6. The variation of stress $\sigma_{\mathrm{x}}$ with the distance to the tip

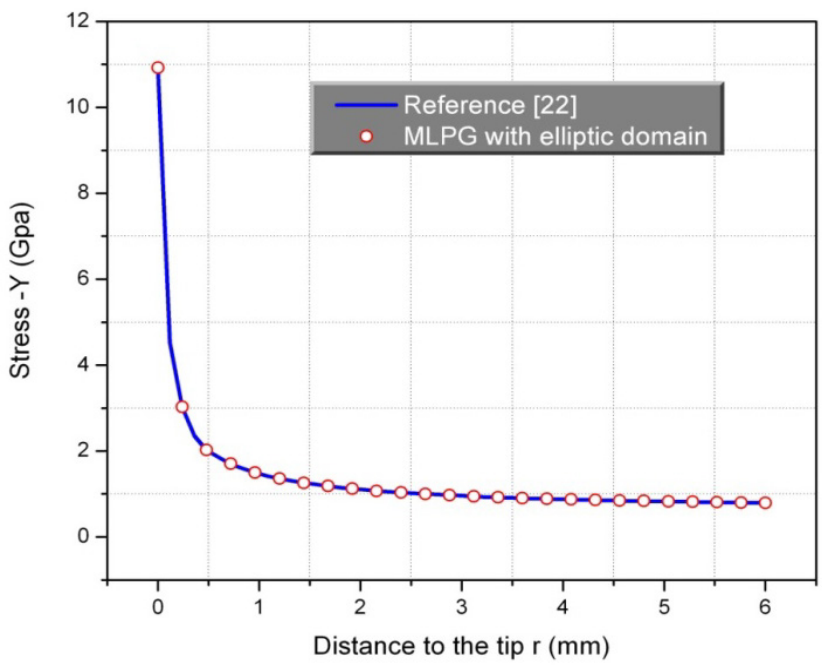

Figure 7. The variation of stress $\sigma_{\mathrm{y}}$ with the distance to the tip

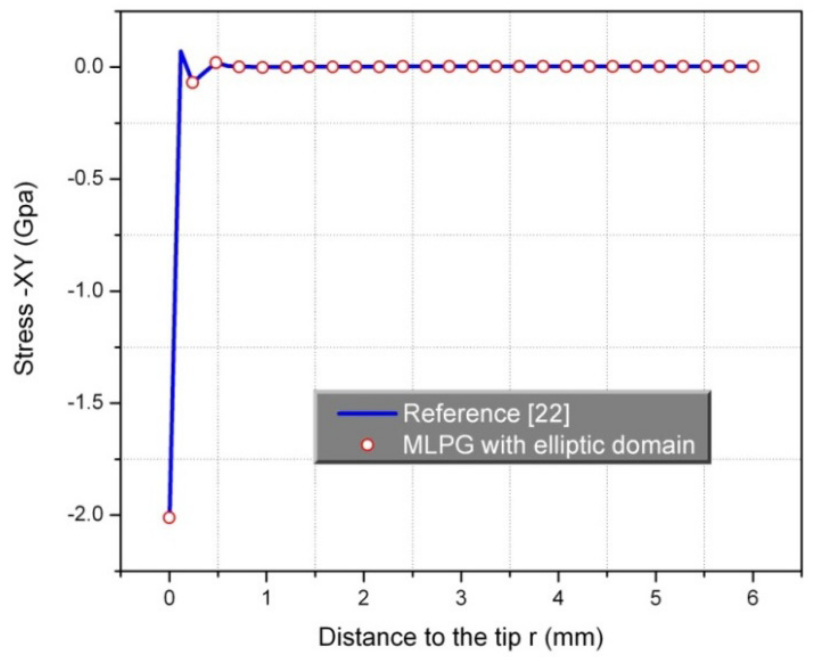

Figure 8. The variation of stress $\sigma_{x y}$ with the distance to the tip

\section{Conclusions}

- Elliptic domain of influence is used local weak form method, particularly in the Meshless Local Petro-Galerkin method MLPG, to study the possibility of using this local domain in the computational mechanics.

- This domain changes the behavior of work of the used weight function in extracting the data from the nodes because in the elliptic support each node has three characteristic indications that are major radius, inner radius, and the direction of major local domain.

- The space that is covered by the elliptic domain is less than the area of the circle domain at the same main diameter, and this reduces the computational time of the required calculation. Thus, using this domain, influence domain of a node is determined by three parameters in contrast to one in the other domains.

- The availability of more controlling parameters for 
determination of the influence domain of each node helps to increase computational efficiency of MLPG method as it clear in reducing the time cost up to $15 \%$ and the results error up to $25 \%$.

- This paper can be extended by changing the direction of major local domain in the calculation or by using in dynamic elasticity problems, as well as in the use of the domain in the other advanced applications.

\section{REFERENCES}

[1] G.R. Liu.,"Meshfree methods-moving beyond finite element method", $2^{\text {nd }}$ edition, Book (2010), CRC Press LLC,

[2] V. P. Nguyena, T. Rabczuk, S. Bordas, and M. Duflot, "Meshless methods: A review and computer implementation aspects", Math. and Comp. in Simulation 79 (2008) 763-813.

[3] Satya N. Atluri , Shengping Shen "The Meshless Local Petrov-Galerkin (MLPG) Method: ASimple \& Less-costly Alternative to the Finite Element and Boundary Element Methods", CMES, vol.3, no.1, pp.11-51, 2002.

[4] S. N. Atluri, J. Sladek, V. Sladek, T. Zhu"The local boundary integral equation (LBIE) and it's meshless implementation for linear elasticity", Computational Mechanics 25 (2000) 180-198.

[5] H. Lin , S.N. Atluri "The Meshless Local Petrov-Galerkin (MLPG) Method for Solving Incompressible Navier-Stokes Equations", CMES, vol.2, no.2, pp.117-142, 2001.

[6] H. Lin, S.N. Atluri "Meshless Local Petrov-Galerkin (MLPG)Method for Convection-Diffusion Problems", CMES, vol.1, no.2, pp.45-60, 2000.

[7] Z. D. Han,H. T. Liu, A. M.Rajendran, S. N. Atluri "The Applications of Meshless Local Petrov-Galerkin (MLPG) Approaches in High-Speed Impact, Penetration and Perforation Problems", CMES, vol.14, no.2, pp.119-128, 2006.

[8] T. Belytschko, Y. Y. Lu, and L., Gu, "Element-Free Galerkin Methods", International Journal for Numerical Methods in Engineering, Vol. 37, pp. 229-256, 1994.

[9] J. Dolbow, and T. Belytschko, "An Introduction to Programming the Meshless Element Free Galerkin Method", Achieves of Computational Mechanics, Vol.15, No. 3, pp. 207-241, 1998.
[10] S. N. Atluri, T. Zhu "New concepts in meshless methods", Int J Numer Methods Eng. 47, 537-556( 2000).

[11] ]S. N. Atluri, T. Zhu " A new Meshless Local Petrov-Galerkin (MLPG) approach in computational mechanics ", Computational Mechanics 22 (1998) 117-127

[12] H.-K. Ching, R. C. Batra " Determination of Crack Tip Fields in Linear Elastostatics by the Meshless Local Petrov-Galerkin (MLPG) Method ", CMES, vol.2, no.2, pp.273-289, 2001.

[13] Z.Tang, S. Shen, S.N. Atluri " Analysis of Materials with Strain-Gradient Effects: A Meshless Local Petrov-Galerkin(MLPG) Approach, with Nodal Displacements only", CMES, vol.4, no.1, pp.177-196, 2003.

[14] J. Sladek1, V. Sladek1, S.N. Atluri " Meshless Local Petrov-Galerkin Method in Anisotropic Elasticity", CMES, vol.6, no.5, pp.477-489, 2004.

[15] S. N. Atluri, T.-L. Zhu" The meshless local Petrov-Galerkin (MLPG) approach for solving problems in elasto-statics", Computational Mechanics 25 (2000) 169-179 (C) Springer-Verlag 2000.

[16] Shengping Shen,S. N. Atluri " Multiscale Simulation Based on The Meshless Local Petrov-Galerkin (MLPG) Method", CMES, vol.5, no.3, pp.235-255, 2004.

[17] Z. D. Han, A.M. Rajendran, S.N. Atluri" Meshless Local Petrov-Galerkin (MLPG) Approaches for Solving Nonlinear Problems with Large Deformations and Rotations", CMES, vol.10, no.1, pp.1-12, 2005.

[18] P. Lancaster and K. Salkauskas "Surfaces Generated by Moving Least Squares Methods",Mathematics of Computation, Vol. 37, pp. 141-158, 1981.

[19] Q. Li , S. Shen, Z. D. Han, and S. N. Atluri " Application of Meshless Local Petrov-Galerkin (MLPG) to Problems with Singularities, and Material Discontinuities, in 3-D Elasticity", CMES, vol.4, no.5, pp.571-585, 2003.

[20] Z. D. Han ,S. N. Atluri "Meshless Local Petrov-Galerkin (MLPG) approaches for solving 3D Problems in elasto-statics", CMES, vol.6, no.2, pp.169-188, 2004.

[21] S. N. Atluri, Z. D. Han1 and A.M. Rajendran," A New Implementation of the Meshless Finite Volume Method, Through the MLPG "Mixed" Approach"CMES: Computer Modeling in Engineering \& Sciences, vol.6, no.6, pp.491-513, 2004

[22] Liu Kaiyuan , Long Shuyao , Li Guangyao "A simple and less-costly meshless local Petrov-Galerkin (MLPG) method for the dynamic fracture problem", Elsevier Ltd, Engineering Analysis with Boundary Elements 30 (2006) 72-76. 